\title{
Genome-wide analysis of the potato Hsp20 gene family: identification, genomic organization and expression profiles in response to heat stress
}

Peng Zhao ${ }^{1 \dagger}$, Dongdong Wang ${ }^{1 \dagger}$, Ruoqiu Wang ${ }^{1}$, Nana Kong ${ }^{1}$, Chao Zhang ${ }^{1}$, Chenghui Yang ${ }^{1}$, Wentao Wu ${ }^{1,2}$, Haoli Ma ${ }^{1 *}$ and Qin Chen ${ }^{1 *}$ (i)

\begin{abstract}
Background: Heat shock proteins (Hsps) are essential components in plant tolerance mechanism under various abiotic stresses. Hsp20 is the major family of heat shock proteins, but little of Hsp20 family is known in potato (Solanum tuberosum), which is an important vegetable crop that is thermosensitive.

Results: To reveal the mechanisms of potato Hsp20s coping with abiotic stresses, analyses of the potato Hsp20 gene family were conducted using bioinformatics-based methods. In total, 48 putative potato Hsp20 genes (StHsp20s) were identified and named according to their chromosomal locations. A sequence analysis revealed that most StHsp20 genes (89.6\%) possessed no, or only one, intron. A phylogenetic analysis indicated that all of the StHsp2O genes, except 10, were grouped into 12 subfamilies. The 48 StHsp20 genes were randomly distributed on 12 chromosomes. Nineteen tandem duplicated StHsp2Os and one pair of segmental duplicated genes (StHsp20-15 and StHsp20-48) were identified. A cis-element analysis inferred that StHsp20s, except for StHsp20-41, possessed at least one stress response cis-element. A heatmap of the StHsp2O gene family showed that the genes, except for StHsp20-2 and StHsp20-45, were expressed in various tissues and organs. Real-time quantitative PCR was used to detect the expression level of StHsp20 genes and demonstrated that the genes responded to multiple abiotic stresses, such as heat, salt or drought stress. The relative expression levels of 14 StHsp20 genes (StHsp20-4, 6, 7, 9, 20, 21, 33, 34, 35, 37, 41, 43, 44 and 46) were significantly up-regulated (more than 100-fold) under heat stress.
\end{abstract}

Conclusions: These results provide valuable information for clarifying the evolutionary relationship of the StHsp20 family and in aiding functional characterization of StHsp2O genes in further research.

Keywords: Genome-wide, Potato, Hsp20 gene family, Heat stress

\section{Background}

Plants live in an open environment and are exposed to various abiotic and biotic stresses. The increased temperatures associated with global warming have adverse effects on plant growth and development [1]. During tuber development, high temperatures can change plant source-sink relationship, which disrupts tuber initiation, and thus reduces yield and quality [2-4]. To survive and

\footnotetext{
* Correspondence: mahaoli@nwsuaf.edu.cn; chenpeter2289@nwsuaf.edu.cn ${ }^{\dagger}$ Equal contributors

${ }^{1}$ State Key Laboratory of Crop Stress Biology for Arid Areas, College of Agronomy, Northwest A \& F University, Yangling, Shaanxi 712100, China Full list of author information is available at the end of the article
}

avoid adverse effects under high temperature, plants established self-defense mechanisms during evolution. Heat shock proteins (Hsps) are a group of proteins synthetized under high temperature that exist in living organisms from bacteria to humans [5]. In plants, the Hsp genes participate in many developmental processes and responding to abiotic stresses [6, 7].

According to previous studies, Hsps can be grouped into five families including Hsp100, Hsp90, Hsp70, Hsp60 and Hsp20 based on their molecular weight and sequence homology $[6,8]$. The molecular weight of Hsp20 protein ranges from 15 to $42 \mathrm{kDa}$, thus is also 
called as small Hsp [9]. Hsp20 is the major family of heat shock proteins induced by elevated temperatureassociated stress in plants [10, 11]. Hsp20 is encoded by a multigene family and is considered the most produced protein under heat stress conditions in many higher plants $[12,13]$.

Hsp20s are ATP-independent molecular chaperones and can form oligomeric protein complexes of 200$800 \mathrm{kDa}$, which consist of 9 to 50 subunits [14, 15]. Hsp20 can avert protein denaturation, and thus maintain the stability and normal functions of proteins in both eukaryotic and prokaryotic cells $[6,16]$. The existing evidence suggests that Hsp20 plays an important role in plant heat tolerance. Hsp20s possess a conserved structure, consisting of a variable $\mathrm{N}$-terminal region, a more conserved $\mathrm{C}$ terminal region and a $\mathrm{C}$-terminal extension [6]. The more conserved $\mathrm{C}$-terminal region is usually named as the alpha-crystallin domain (ACD), which contains approximately 80 to 100 amino acid residues. The three different regions possess varied functions. The $\mathrm{ACD}$ functions in substrate interactions, while the $\mathrm{N}$-terminal region participates in substrate binding and the $\mathrm{C}$-terminal extension is responsible for homo-oligomerization [17-20]. The ACD contains two conserved regions, one in the N-terminal consensus region and the other is connected through a hydrophobic $\beta 6$-loop at the $\mathrm{C}$-terminal common region. The two conserved regions consist of 4 anti-parallel sheets and $3 \beta$-strands respectively $[16,21]$. Furthermore, unlike other $H s p$ families, the $H s p 20$ gene family exhibits extensive sequence variability and evolutionary divergence [22].

The number of plant $H s p 20$ genes is approximately four times greater than that of animals [10]. The Hsp20 gene family members have been investigated in many plants, such as Arabidopsis, rice, soybean, pepper and tomato. There are $19 \mathrm{Hsp} 20$ genes in Arabidopsis [23], 39 in rice [24], 51 in soybean [25], 35 in pepper [26] and 42 in tomato [27]. Following maize, wheat and rice, potato is the fourth-largest food crop in the world. Potatoes are formed from underground stems through a process known as tuberization, but high temperatures inhibit the process and decrease the amount of photosynthetic product transported into the tubers, causing a large yield loss [28]. To date, the potato Hsp2O gene family members have not been identified and their functions under heat stress conditions remain to be elucidated. With the availability of the whole-genome sequence of potato, it is now possible to more fully study the potato Hsp2O gene family.

Here, we used bioinformatics methods to identify $H s p 20$ genes from potato genome, and analyze the sequence features, chromosomal locations, phylogenetic relationships, cis-elements, tissue-specific expression levels and dynamic expression patterns in response to different abiotic stresses, including heat stress. The results provide useful information for further functional investigations of the $S t H s p 20$ gene family.

\section{Methods \\ Identification of the $\mathrm{Hsp} 20$ family members in potato genome}

The whole potato protein sequence was downloaded from the Potato Genome Sequencing Consortium (PGSC, http://potato.plantbiology.msu.edu/integrated_searches.shtml). To identify potato Hsp20 candidates, the Hidden Markov Model (HMM) analysis was used for the search. We downloaded HMM profile of Hsp20 (PF00011) from Pfam protein family database (http:// pfam.xfam.org/) and used it as the query $(P<0.001)$ to search the potato protein sequence data [29]. To avoid missing probable Hsp20 members because of incomplete ACD domains, a BLASTP-algorithm based search using Arabidopsis Hsp20 amino acid sequences as queries was conducted with an e-value $\leq 1 \mathrm{e}^{-3}$. Additionally, keywords "Hsp20" and "small heat shock protein" were employed to search against PGSC database. After removing all of the redundant sequences, the output putative Hsp20 protein sequences were submitted to CDD (https:// www.ncbi.nlm.nih.gov/Structure/bwrpsb/bwrpsb.cgi), Pfam and SMART (http://smart.embl-heidelberg.de/) to confirm the conserved Hsp20 domain. The predicted protein sequences lacking the Hsp20 domain or with a molecular weight outside of the 15-42-kDa range were excluded. All of the non-redundant and high-confidence genes were assigned as potato Hsp20s (StHsp20s). These StHsp2O genes were named on the basis of their positions on pseudomolecules [24].

\section{Sequence analysis and structural characterization}

All of the high-confidence Hsp20 sequences were submitted to ExPASy (http://web.expasy.org/protparam/) to calculate the number of amino acids, molecular weights and theoretical isoelectric points (pI). The chromosomal locations and intron numbers of $S t H s p 20$ s were acquired through the PGSC. The MEME program (version 4.11.2, http://alternate.meme-suite.org/tools/meme) was used to identify the conserved motifs in the StHsp20s sequences, with the following parameters: any number of repetitions, maximum of 10 misfits and an optimum motif width of 6 - 200 amino acid residues. The exon-intron structures of the StHsp2O genes were identified on the Gene Structure Display Server (GSDS, http://gsds.cbi.pku.edu.cn/) [30].

\section{Chromosomal localization and gene duplication}

The chromosomal positions of the StHsp2O genes were acquired from the potato genome browser at the PGSC. MapChart software [31] was used for the mapping of 
StHsp20 genes' chromosomal positions and relative distances. The StHsp2O gene duplication was confirmed based on two criteria: (a) the length of the shorter aligned sequence covered $>70 \%$ of the longer sequence; and (b) the similarity of the two aligned sequences were $>70 \%[32,33]$. Two genes separated by five or fewer genes in 100-kb chromosome fragment were considered as tandem duplicated genes [34]. The segmental duplicated genes of StHsp 20 were identified by searching the segmental genome duplications of potato at the Plant Genome Duplication Database (PGDD, http://chibba.agtec.uga.edu/duplication/).

\section{Phylogenetic analysis and classification of potato Hsp20 genes}

The full-length amino acid sequences of Hsp20s (Additional file 1: Table S1) derived from Arabidopsis [35], soybean [25], rice [24] and Populus [36] combined with newly identified StHsp20s were used for phylogenetic analysis. All of the acquired sequences were first aligned by ClustalX (version 1.83) software [37] with the default parameters. An unrooted neighbor-joining phylogenetic tree was constructed using MEGA6 software [38] with bootstrap test of 1000 times. The potato Hsp20 genes were classified into different groups according to the topology of phylogenetic tree and the classifications of Hsp2Os in four other species.

\section{Analysis of Cis-acting element in StHsp20 genes' promoters}

The upstream sequences $(1.5 \mathrm{~kb})$ of the StHsp2O-coding sequences were retrieved from the PGSC and then submitted to PlantCARE (http://bioinformatics.psb.ugent.be/ webtools/plantcare/html/; [39]) to identify six regulatory elements, abscisic acid (ABA)-responsive elements, involved in the ABA responsiveness; dehydration-responsive elements (DREs), involved in dehydration, low-temp and salt stresses; heat stress elements (HSEs), involved in heat stress response; low temperature responsive elements (LTRE), involved in low-temperature response; TC-rich repeats, involved in defense and stress response; and Wboxes, binding site of WRKY transcription factor in defense responses.

\section{Plant materials and abiotic stress treatments}

The doubled monoploid (DM) potato was used in this study. All of the lines were cultured in Murashige and Skoog (MS) medium [40] containing 3\% sucrose and $0.8 \%$ agar at $\mathrm{pH}$ 5.9. The plant material was sustained in an artificial climate chamber with $16 \mathrm{~h}$ light $/ 8 \mathrm{~h}$ dark photoperiod and temperature of $22 \pm 1{ }^{\circ} \mathrm{C}$. The fourweek-old plantlets were then transferred into cuvettes containing 1/2 MS liquid medium and maintained in an artificial growth chamber at $22 \pm 1{ }^{\circ} \mathrm{C}(16 \mathrm{~h}$ light $/ 8 \mathrm{~h}$ dark period) for a week before being subjected to an abiotic stress. For heat stress, the plantlets were exposed to $35{ }^{\circ} \mathrm{C}$; for salt stress, the plantlets were incubated with $150 \mathrm{mM} \mathrm{NaCl}$; and for drought stress, the plantlets were treated with $260 \mathrm{mM}$ mannitol. Under these different stress conditions, the aboveground of whole plants were collected at 0,3 and $24 \mathrm{~h}$ after treatments. All of the collected samples were froze in liquid nitrogen rapidly and stored at $-80{ }^{\circ} \mathrm{C}$ refrigerator before RNA extraction.

RNA-sequencing (RNA-seq) data analysis of StHsp20 genes The Illumina RNA-seq data were downloaded from the PGSC to study the expression patterns of StHsp2O genes. The RNA-seq data (Additional file 2: Table S2) included various developmental stages, tissues and stress treatments. To render the data suitable for cluster displays, absolute FPKM values were divided by the mean of all of the values, and the ratios were transformed by $\log 2$. HemI [41] software was used to generate the heatmap.

\section{Total RNA extractions and expression analyses of potato Hsp20 genes}

Primer Premier 5 was used to design primers specific to the StHsp20 genes (Additional file 3: Table S3). Total RNA was extracted using an RNAsimple Total RNA Kit (BioTeke, Beijing, China). The cDNA was reversetranscribed by First Strand cDNA Synthesis Kit, ReverTra Ace- $\alpha$ (TOYOBO, Shanghai, China). All of the operational procedures followed the manufacturer's protocols. Before the qRT-PCR analysis, $1 \mu \mathrm{l}$ cDNA was diluted with $4 \mu \mathrm{l}$ nuclease-free water.

qRT-PCR was carried out using the KAPA SYBR FAST qPCR Kit Master Mix $(2 \times)$ Universal (KAPA BIOSYSTEMS, Boston, United States) on a Bio-Rad CFX96 Real Time PCR System. Each PCR reaction was conducted in a $20-\mu$ reaction volume containing $10 \mu \mathrm{l}$ KAPA SYBR, $0.5 \mu \mathrm{l} 10 \mu \mathrm{M}$ solution of each primer, $1 \mu \mathrm{l}$ diluted cDNA

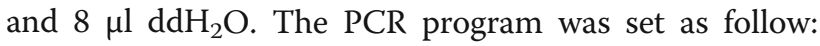
$95{ }^{\circ} \mathrm{C}$ for $2 \mathrm{~min}$ and 40 cycles of $95^{\circ} \mathrm{C}$ for $5 \mathrm{~s}$ and $60{ }^{\circ} \mathrm{C}$ for $30 \mathrm{~s}$. The melt curve was analyzed from $65^{\circ} \mathrm{C}$ to $95^{\circ}$ $\mathrm{C}$ with increments of $0.5{ }^{\circ} \mathrm{C}$ every $5 \mathrm{~s}$. For each sample, three biological repeats, with two technical replicates each, were performed to acquire reliable results. The housekeeping gene ef $1 \alpha$ was used as the internal reference gene. The synthetic cDNA was diluted to 3-, 9-, 27- and 81-fold to establish the standard curve for each StHsp 20 gene and ef1 $\alpha$. The relative expression levels of the StHsp2O genes were calculated using the standard curve and normalized by the control's expression. The results were displayed by means \pm standard deviation (SD). 


\section{Results}

Identification and analysis of StHsp20 genes

A total of $58 \mathrm{Hsp} 20$ s were obtained by HMM analysis, 52 sequences were found by local BLASTP, and 35 sequences were acquired by keyword search against the PGSC database. After removing the repetitive sequences, 65 sequences were reserved and submitted to CDD, Pfam and SMART to confirm the ACD domain. Sequences without a typical ACD domain and with a molecular weight outside of the $15-42-\mathrm{kDa}$ range were excluded. Finally, 48 sequences were confirmed as potato Hsp20 genes and named based on their chromosomal locations. Gene names, gene IDs, chromosomal locations, open reading frame lengths, exon numbers, amino acid numbers, molecular weights and pIs were listed in Table 1. The lengths of the StHsp20 proteins ranged from 133 (StHsp20-36) to 303 amino acids (StHsp20-15). The molecular weights of StHsp20s were between $15.3 \mathrm{kDa}$ (StHsp20-36) and 34.0 kDa (StHsp2015). StHsp20 genes were distributed on 12 potato chromosomes. The predicted pI values of StHsp20 ranged from 4.91 (StHsp20-5) to 9.88 (StHsp20-39).

\section{StHsp20 gene structure}

Structures and phases of introns/exons were determined by the alignment of genomic DNA with full-length cDNA of StHsp20s. Among the StHsp20 genes, nearly half $(20,41.7 \%)$ were intronless, $23(47.9 \%)$ had one intron, and only 5 genes (10.4\%), StHsp20-15 (12 introns), StHsp20-22 (5 introns), StHsp20-15 (12 introns), StHsp20-45 (8 introns) and StHsp20-48 (5 introns), had two or more introns (Fig. 1). Interestingly, all of the tandem duplicated genes were intronless and the pair of segmentally duplicated genes, StHsp15 and StHsp48, had multiple introns. StHsp 48 was shorter than StHsp15 in sequence length, but shared a highly conserved region with StHsp15. The conserved region possessed the same intron phase $(1,2,0,0$ and 0$)$. The result suggested a particular phylogenetic relationship between the two segmentally duplicated genes.

The conserved motifs of StHsp20 proteins were identified by MEME website, and 10 were found. The lengths of these conserved motifs varied from 8 to 113 amino acids. Details of the 10 putative motifs are outlined in Table 2. Based on analyses of Pfam, CDD and SMART, Motif 1 completely corresponded to the region of the conserved ACD. The full sequences of Motifs 2, 3 and 7 together formed a highly conserved complete ACD. The majority of the StHsp20 proteins (58.3\%) contained Motif 1 or the combination of Motifs 2, 3 and 7. Other StHsp20 proteins lacked the complete combination of motifs. StHsp20-1, 2, 3, 8, 31, 40 and 42 contained Motif 8 , which was predicted to be a transmembrane region. Ten StHsp20 proteins could not be classified with other types of StHsp20 proteins (Fig. 2). The different compositions of the ACD domain may indicate functional diversity. The same group of StHsp20 proteins in the phylogenetic tree shared common motifs and indicated they were highly conserved.

\section{Phylogenetic analysis of StHsp20 genes}

To analyze the evolutionary relationships of $H s p 20$ genes in potato, Arabidopsis, soybean, rice and Populus, an unrooted phylogenetic tree was constructed using fulllength amino acid sequences. In total, 19 sequences from Arabidopsis, 22 sequences from rice, 47 sequences from potato, 46 sequences from soybean and 25 sequences from Populus were assessed in the phylogenetic tree (Fig. 2). The potato Hsp20 family member StHsp20-29 was excluded from the phylogenetic tree because it was too divergent to be aligned with other sequences. The $159 \mathrm{Hsp} 20$ s were classified into 12 distinct subfamilies, 71 cytosol Is (CIs), 13 CIIs, 11 CIIIs, 3 CIVs, 5 CVs, 3 CVIs, 3 CVIIs, 5 mitochondria Is (MIs), 6 MIIs, 12 plastids (Ps), 6 peroxisomes (Pos) and 11 endoplasmic reticulum (ERs). However, the remaining 10 potato Hsp20s could not be clustered into any subfamily. Except for the unclassified StHsp20s, 37 StHsp20s existed in 11 subfamilies, except for the CIV subfamily. Most of the Hsp20s, including 29 StHsp20s, were classified into CI-CVII, which indicated that cytosol might be the main functional area for plant Hsp20s. Remarkably, StHsp20 members were more closely related to those in the same subfamily from different species than to the other Hsp20s from the same species, which implied a relatively high synteny between the same Hsp20 subfamily across various species. It was interesting that the $\mathrm{P}$ and M (MI and MII) subfamily members had a close relationship with each other, which indicated that the $M$ subfamily evolved from the P subfamily once again [6]. No Hsp20 protein of monocotyledon (rice) was found in CIV subfamily. According to previous study [35], CIV subfamily of Hsp20s existed only in dicotyledon.

A close relationship between the phylogenetic classification and intron pattern existed. According to previous research, three patterns were proposed. Pattern 1 means no intron, Pattern 2 means one intron, and Pattern 3 means more than one intron [24]. Most StHsp20 members of the CI subfamily lacked introns, and the CII and ER subfamilies had no introns. However, all of the members of the CV, CVI, CVII, Po, MI and MII subfamilies had one intron, which indicated a close phylogenetic relationship (Fig. 1; Table 1). In addition, three genes (StHsp15, StHsp45 and StHsp48) belonging to the CIII subfamily had 12, 8 and 5 introns, respectively (Fig. 1; Table 1). The presence of multiple introns indicated a particular phylogenetic status. 
Table 1 Features of StHsp20 genes identified in potato

\begin{tabular}{|c|c|c|c|c|c|c|c|c|}
\hline Name & Gene ID & Chr. & Genomic Location & ORF & Exon & $\mathrm{AA}$ & $\mathrm{MW}(\mathrm{kDa})$ & $\mathrm{pl}$ \\
\hline StHsp20-1 & PGSC0003DMG400008713 & 1 & $6195606-6196992$ & 732 & 2 & 243 & 26.8 & 5.80 \\
\hline StHsp20-2 & PGSC0003DMG400008714 & 1 & $6199128-6200118$ & 669 & 2 & 222 & 24.5 & 8.91 \\
\hline StHsp20-3 & PGSC0003DMG400008715 & 1 & $6205818-6207478$ & 639 & 2 & 212 & 23.8 & 9.30 \\
\hline StHsp20-4 & PGSC0003DMG400020718 & 1 & $79702817-79703750$ & 576 & 1 & 191 & 21.8 & 6.87 \\
\hline StHsp20-5 & PGSC0003DMG400016460 & 2 & $36223235-36224237$ & 414 & 2 & 137 & 15.7 & 4.91 \\
\hline StHsp20-6 & PGSC0003DMG400012619 & 2 & $47595303-47596399$ & 426 & 2 & 141 & 16.3 & 8.32 \\
\hline StHsp20-7 & PGSC0003DMG400003219 & 3 & $46254502-46257461$ & 702 & 2 & 233 & 25.9 & 6.98 \\
\hline StHsp20-8 & PGSC0003DMG400025350 & 3 & $53138385-53139192$ & 651 & 2 & 216 & 24.5 & 4.98 \\
\hline StHsp20-9 & PGSC0003DMG400024476 & 3 & $53878612-53879610$ & 576 & 1 & 191 & 21.9 & 6.87 \\
\hline StHsp20-10 & PGSC0003DMG400009173 & 3 & $61718867-61719420$ & 444 & 2 & 147 & 16.5 & 7.64 \\
\hline StHsp20-11 & PGSC0003DMG400023622 & 4 & $8358204-8359541$ & 438 & 2 & 145 & 16.1 & 6.92 \\
\hline StHsp20-12 & PGSC0003DMG400024099 & 4 & $60045801-60046965$ & 882 & 2 & 293 & 33.1 & 6.00 \\
\hline StHsp20-13 & PGSC0003DMG400031133 & 4 & $61728105-61729376$ & 510 & 2 & 169 & 18.6 & 5.30 \\
\hline StHsp20-14 & PGSC0003DMG400009996 & 4 & $71887737-71891840$ & 462 & 2 & 153 & 16.7 & 7.71 \\
\hline StHsp20-15 & PGSC0003DMG400010001 & 4 & 71971113 - 71980699 & 912 & 13 & 303 & 34.0 & 9.68 \\
\hline StHsp20-16 & PGSC0003DMG400011977 & 5 & $11646812-11649618$ & 681 & 3 & 226 & 26.2 & 9.39 \\
\hline StHsp20-17 & PGSC0003DMG400030427 & 6 & $56896895-56897612$ & 465 & 1 & 154 & 17.7 & 5.57 \\
\hline StHsp20-18 & PGSC0003DMG400030426 & 6 & $56893292-56894077$ & 465 & 1 & 154 & 17.6 & 5.83 \\
\hline StHsp20-19 & PGSC0003DMG400030339 & 6 & $56900911-56901677$ & 465 & 1 & 154 & 17.7 & 6.20 \\
\hline StHsp20-20 & PGSC0003DMG400030340 & 6 & $56905147-56905872$ & 465 & 1 & 154 & 17.6 & 7.91 \\
\hline StHsp20-21 & PGSC0003DMG400030341 & 6 & $56907909-56908742$ & 465 & 1 & 154 & 17.6 & 5.57 \\
\hline StHsp20-22 & PGSC0003DMG401017288 & 7 & $50794176-50799268$ & 753 & 6 & 250 & 27.9 & 9.19 \\
\hline StHsp20-23 & PGSC0003DMG400019265 & 7 & $54203551-54205245$ & 573 & 2 & 190 & 21.8 & 5.49 \\
\hline StHsp20-24 & PGSC0003DMG400021737 & 8 & $34366816-34367720$ & 477 & 1 & 158 & 17.7 & 6.17 \\
\hline StHsp20-25 & PGSC0003DMG400008187 & 8 & $34544813-34545655$ & 477 & 1 & 158 & 17.6 & 6.17 \\
\hline StHsp20-26 & PGSC0003DMG400004808 & 8 & $52375001-52376265$ & 636 & 2 & 211 & 23.9 & 6.45 \\
\hline StHsp20-27 & PGSC0003DMG400004807 & 8 & $52380425-52381599$ & 588 & 2 & 195 & 21.4 & 8.65 \\
\hline StHsp20-28 & PGSC0003DMG400004806 & 8 & $52390914-52392554$ & 513 & 2 & 170 & 18.5 & 5.05 \\
\hline StHsp20-29 & PGSC0003DMG400020341 & 9 & $829873-831558$ & 486 & 2 & 161 & 19.2 & 9.77 \\
\hline StHsp20-30 & PGSC0003DMG400011719 & 9 & $888985-890624$ & 582 & 2 & 193 & 22.7 & 7.06 \\
\hline StHsp20-31 & PGSC0003DMG400002009 & 9 & $6945529-6946992$ & 744 & 2 & 247 & 27.5 & 5.53 \\
\hline StHsp20-32 & PGSC0003DMG400011628 & 9 & $11636814-11637710$ & 465 & 1 & 154 & 17.5 & 6.21 \\
\hline StHsp20-33 & PGSC0003DMG400011630 & 9 & $11675983-11676827$ & 495 & 1 & 164 & 18.8 & 6.15 \\
\hline StHsp20-34 & PGSC0003DMG400011631 & 9 & $11678942-11679778$ & 474 & 1 & 157 & 17.9 & 6.21 \\
\hline StHsp20-35 & PGSC0003DMG400011632 & 9 & $11684152-11685032$ & 465 & 1 & 154 & 17.5 & 6.21 \\
\hline StHsp20-36 & PGSC0003DMG400014956 & 9 & $31436185-31436781$ & 402 & 1 & 133 & 15.3 & 6.19 \\
\hline StHsp20-37 & PGSC0003DMG400017098 & 9 & $31745004-31745667$ & 423 & 1 & 140 & 16.2 & 5.63 \\
\hline StHsp20-38 & PGSC0003DMG400019136 & 10 & $50699792-50700705$ & 705 & 1 & 234 & 27.3 & 9.56 \\
\hline StHsp20-39 & PGSC0003DMG400019137 & 10 & $50704357-50705144$ & 588 & 1 & 195 & 22.5 & 9.88 \\
\hline StHsp20-40 & PGSC0003DMG400007210 & 10 & $59676867-59677589$ & 444 & 2 & 147 & 16.5 & 8.89 \\
\hline StHsp20-41 & PGSC0003DMG400009255 & 11 & $13518704-13519520$ & 594 & 1 & 197 & 22.4 & 5.41 \\
\hline StHsp20-42 & PGSC0003DMG400018717 & 11 & $42985862-42987493$ & 744 & 2 & 247 & 27.5 & 8.32 \\
\hline StHsp20-43 & PGSC0003DMG400002928 & 12 & $3139670-3140616$ & 468 & 1 & 155 & 17.7 & 5.27 \\
\hline StHsp20-44 & PGSC0003DMG400039484 & 12 & $3142147-3142608$ & 462 & 1 & 153 & 17.3 & 5.54 \\
\hline
\end{tabular}


Table 1 Features of StHsp20 genes identified in potato (Continued)

\begin{tabular}{|c|c|c|c|c|c|c|c|c|}
\hline Name & Gene ID & Chr. & Genomic Location & ORF & Exon & AA & $\mathrm{MW}(\mathrm{kDa})$ & $\mathrm{pl}$ \\
\hline StHsp20-45 & PGSC0003DMG400047019 & 12 & $19504973-19513520$ & 909 & 9 & 302 & 33.8 & 8.24 \\
\hline StHsp20-46 & PGSC0003DMG400028624 & 12 & $49808899-49810200$ & 690 & 2 & 229 & 26.2 & 8.44 \\
\hline StHsp20-47 & PGSC0003DMG402010796 & 12 & $54506438-54507366$ & 750 & 2 & 249 & 28.2 & 8.23 \\
\hline StHsp20-48 & PGSC0003DMG400029311 & 12 & $57570831-57572324$ & 423 & 6 & 140 & 15.5 & 6.19 \\
\hline
\end{tabular}

Chromosomal location and gene duplication of StHsp20s The 48 StHsp 20 genes were distributed on 12 potato chromosomes randomly (Fig. 3). The majority of StHsp20 genes were located on the proximate or the distal ends of the chromosomes. The maximum number of nine predicted $S t H s p 20$ genes, scattered in two clusters, were present on chromosome 9, and only one gene existed on chromosome 5 .

During the progress of evolution, both tandem duplication and segmental duplication contribute to the generation of gene family [42]. Thus, we analyzed the duplication events of StHsp2O genes. Based on the

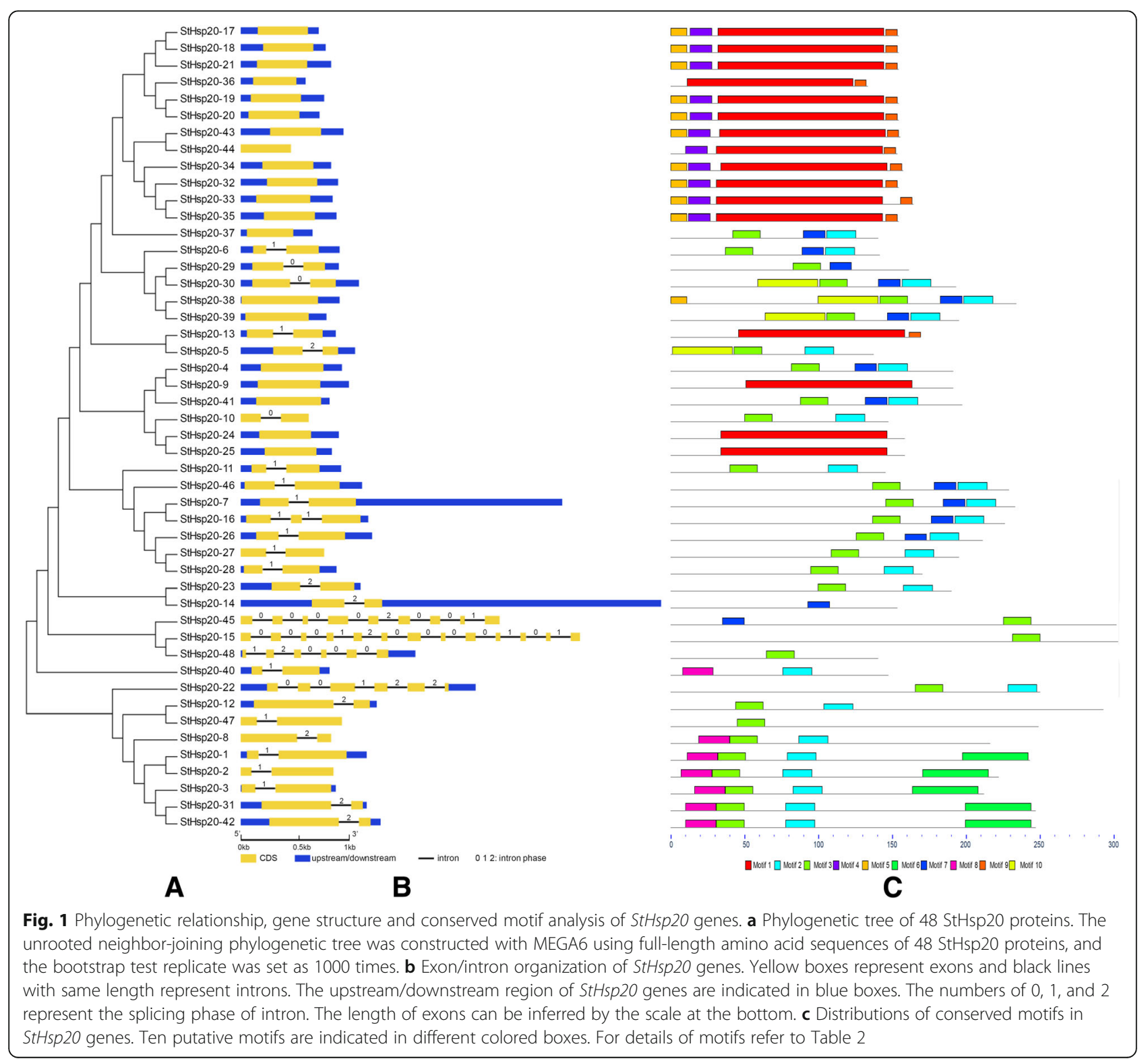


Table $\mathbf{2}$ List of the putative motifs of StHsp20 proteins

\begin{tabular}{|c|c|c|}
\hline Motif & Width & Best possible match \\
\hline 1 & 113 & $\begin{array}{l}\text { FPPSSSRETSAFANTRIDWKET } \\
\text { PEAHVFKVDVPGLKKEEVKVE } \\
\text { VEEDRVLQISGERSREKEEKND } \\
\text { KWHRVERSSGKFMRRFRLPEN } \\
\text { AKMDQIKASMENGVLTVTVP } \\
\text { KEEEKKP }\end{array}$ \\
\hline 2 & 20 & DVDKIKAKMENGVLTVTVPK \\
\hline 3 & 19 & ADLPGLKKEDVKVQVEDNG \\
\hline 4 & 15 & SNIFDPFSLDVFDPF \\
\hline 5 & 11 & MSLIPRFFGGR \\
\hline 6 & 45 & $\begin{array}{l}\text { TDDATIGTASVLAAKLKMPRKVM } \\
\text { NMTLVALLVLGIGLWSNKMKS }\end{array}$ \\
\hline 7 & 15 & RSYGKFSTSFNLPEN \\
\hline 8 & 21 & VYEDFVPSTELVQEEDSDTLL \\
\hline 9 & 8 & VKSIDISG \\
\hline 10 & 41 & $\begin{array}{l}\text { PSHEFYLETPRSLIAPSLSFPHVP } \\
\text { QYMAQIEYKETPEAHIF }\end{array}$ \\
\hline
\end{tabular}

defined criteria, 19 genes (39.6\%) were confirmed to be tandem duplicated genes. Two separate pairs of tandem duplicated genes located on chromosome 10 and chromosome 12. Two groups of three tandem duplicated genes located on chromosome 1 and 8. Five and four tandem duplicated genes located on chromosome 6 and 9, separately. Additionally, two genes (StHsp20-15 and StHsp20-48) were segmentally duplicated genes, and the length of segmentally duplicated chromosome was $625 \mathrm{~kb}$. Segmental duplication only accounted for $4.2 \%$ of the StHsp 20 genes. Based on above results, it could be inferred that tandem duplication and segmental duplication contribute to the expansion of StHsp2O family together, but the former played a predominant role.

\section{Stress-related cis-elements in StHsp20 promoters}

To further study the potential regulatory mechanisms of StHsp20 during abiotic stress responses, the 1.5-kb upstream sequences from the translation start sites of StHsp20 genes (promoter regions of StHsp20-2, StHsp20-11, StHsp20-15 and StHsp20-32 were absent)

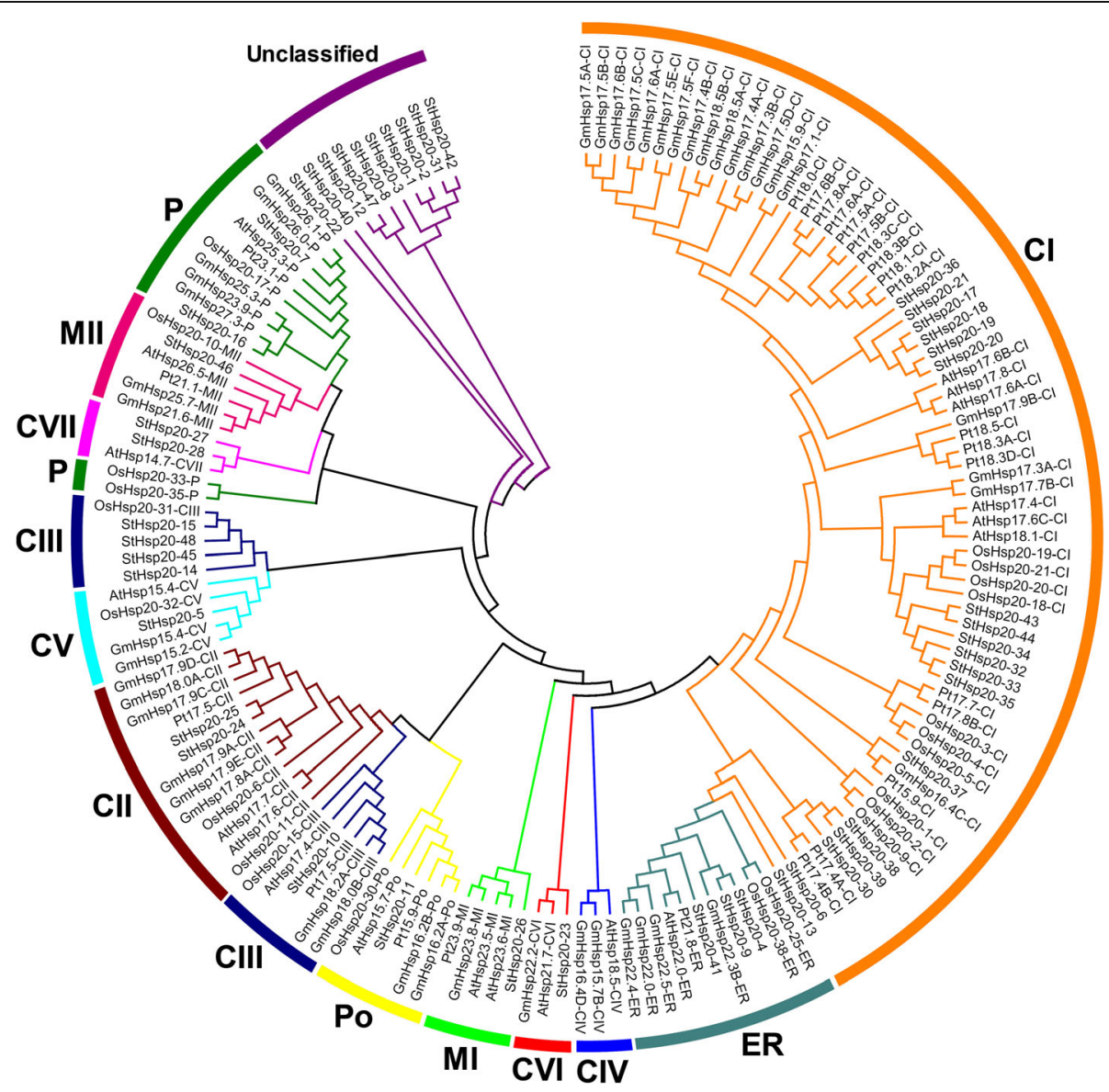

Fig. 2 Phylogenetic tree of Hsp20s from Arabidopsis, Populus, soybean, rice and potatoThe phylogenetic tree was constructed using the NJ (Neighborjoining) method with 1000 bootstrap replications. The 12 subfamilies were distinguished in different colors, and the unclassified StHsp20s were colored in purple 


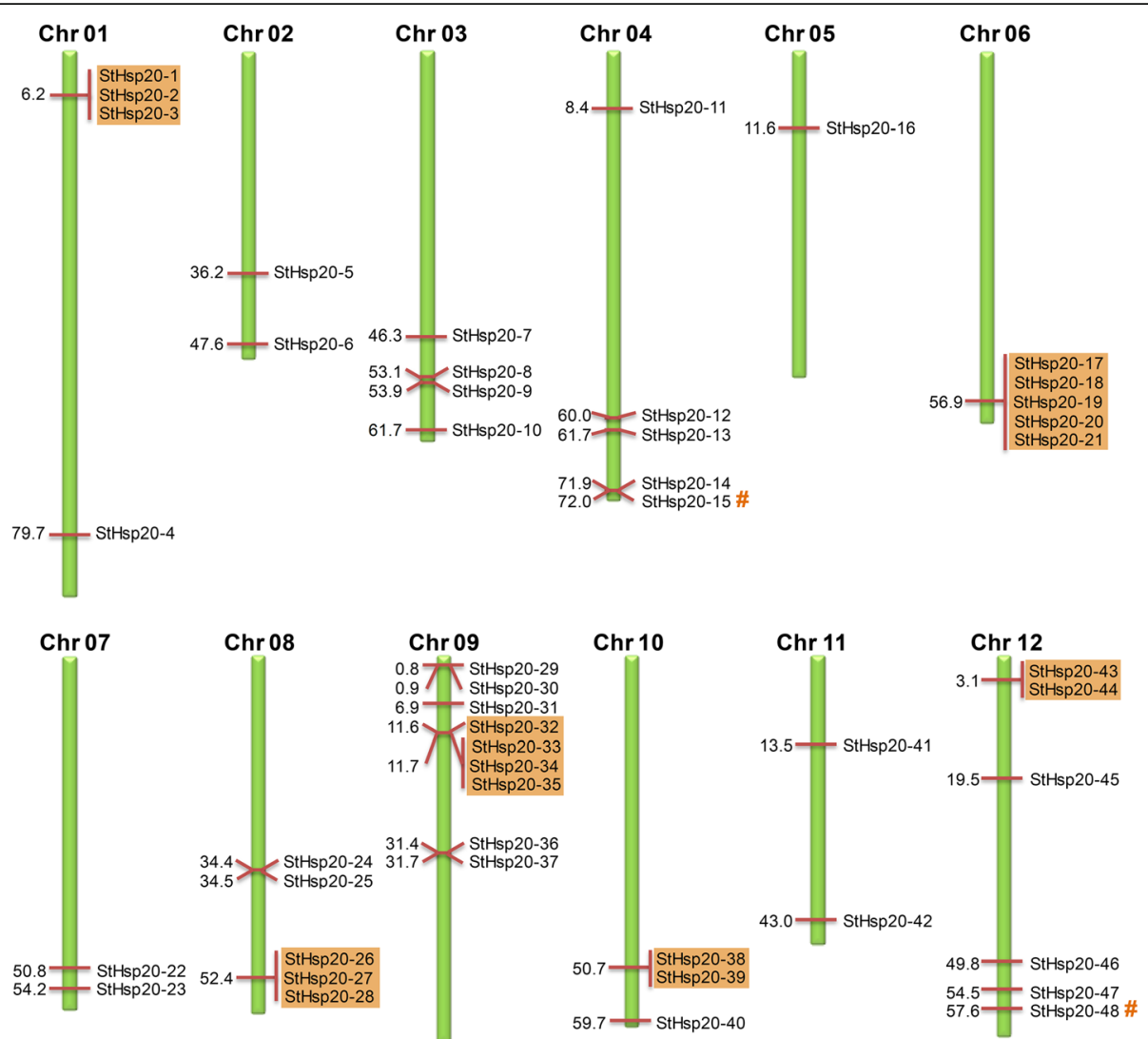

Fig. 3 Chromosomal location and gene duplication of StHsp20s. The tandem duplicated genes are marked by orange rectangles and segmentally duplicated genes are indicated by symbol \#

were submitted into PlantCARE to detect the cis-elements. Six abiotic stress response elements, ABAresponsive elements, DRE, HSE, LTRE, TC-rich repeat and W-box, were analyzed and displayed in Fig. 4. Except for StHsp20-23 and StHsp20-41, the other StHsp20s possessed at least 1 stress-response-related ciselement, which indicated that the expressions of StHsp20s were associated with these abiotic stresses. In total, 32 StHsp20s (72.8\%) had one or more HSEs, suggesting a potential heat-stress response under high temperature conditions. One to two LTREs existed in 11 StHsp20s, and 1 DRE was found in StHsp20-33. TC-rich repeats and W-boxes were located in 34 and 13 StHsp20s, respectively. Anyhow, the cis-element analysis illustrated that $S t H s p 2 O$ genes could respond to different abiotic stresses.

\section{Expression patterns of StHsp20 genes in different tissues} Using the RNA-seq data, a heatmap of $48 \mathrm{StHsp20}$ genes, represented by FPKM values in different tissues and organs, was established by HemI (Fig. 5). Most of StHsp2Os were expressed in one tissue at least, except for StHsp20-2 and $S t H s p 20-45$, which were barely expressed in any tissue or organ. Six genes including StHsp20-18, StHsp20-24,
StHsp20-25, StHsp20-26, StHsp20-29 and StHsp20-30, were highly expressed in all of the tissues. Some StHsp2O genes showed similar expression patterns in various tissues. StHsp20-1, StHsp20-3, StHsp20-12, StHsp20-40 and StHsp20-48 showed relatively high expression levels in vegetative organs, such as shoots, stolons and petioles, but undetectable levels in leaves, sepals, stamens, flowers and petals. StHsp20-6, StHsp20-9, StHsp20-19, StHsp20-33, StHsp20-34, StHsp20-35, StHsp20-41 and StHsp20-43 were highly expressed in callus. StHsp20-7, StHsp20-8, StHsp20-11, StHsp20-27 and StHsp20-32 exhibited high expression levels in shoots and callus.

\section{Expression profiles of StHsp2Os under abiotic stress}

To further explore the expression changes in the StHsp 20 genes under various abiotic stresses including heat, salt and drought, qRT-PCR was used to investigate the transcript levels of each StHsp2O gene with 3 biological repetitions and 2 technical repetitions. Generally, the relative expression level of the $S t H s p 2 O$ genes under all stress conditions fluctuated during the 24-h treatments (Fig. 6). The relative expression level of StHsp2O45 was not shown because the non-specific primers may lead to unreliable results. Most of the StHsp2O genes 


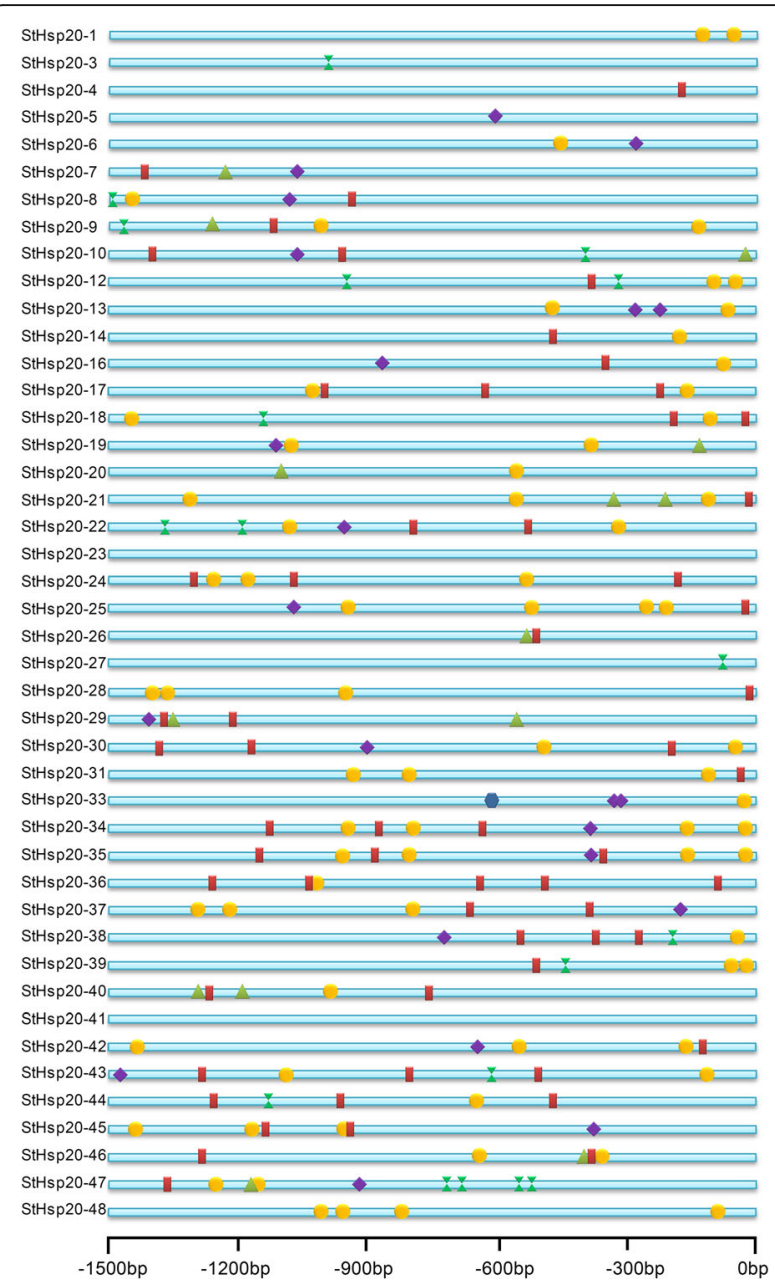

Fig. 4 Predicted cis-elements in StHsp20 promoters. Promoter sequences (-1500 bp) of $44 \mathrm{StHsp20}$ genes (promoter regions of StHsp20-2, StHsp20-11, StHsp20-15 and StHsp20-32 were absent) are analyzed by PlantCARE. The upstream length to the translation start site can be inferred according to the scale at the bottom

were sensitive to heat stress, and none of the genes were down-regulated, but StHsp20-29 and StHsp20-30 showed no differences after being treated for $3 \mathrm{~h}$ and $24 \mathrm{~h}$ under heat stress. The expression levels of StHsp2O-10 and StHsp20-13 were up-regulated only after a 24-h heat treatment. The relative expression levels of 14 StHsp2O genes (StHsp20-4, 6, 7, 9, 20, 21, 33, 34, 35, 37, 41, 43, 44 and 46 ) were extremely up-regulated (more than 100fold) under heat stress compared with the control.

Although the $H s p 2 O$ family is generally induced by heat stress, we also determined whether the family is involved in responses to salt and drought stresses. The expression levels of StHsp2O genes under salt and drought stresses varied among the 47 members. The expression pattern of each StHsp2O was different from that under heat stress. Nearly half of the StHsp2O (40.4\%) genes were down-regulated after

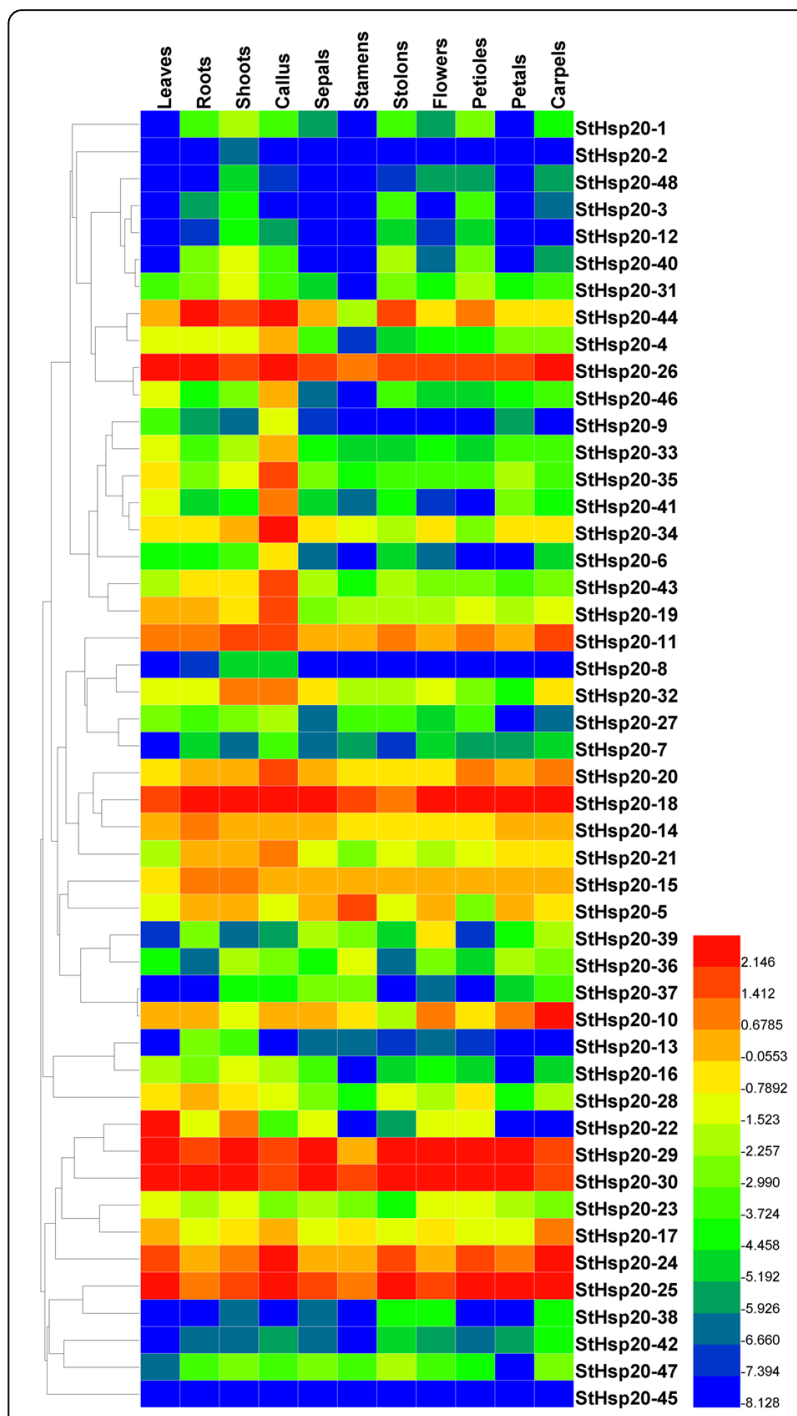

Fig. 5 Expression profiles of StHsp20s in different tissues and organs. FPKM values of StHsp20 genes were transformed by log2 and the heatmap was constructed by Heml software

being treated for $3 \mathrm{~h}$ or $24 \mathrm{~h}$. Six genes (StHsp20-11, 14, 15, 23, 30 and 40) and 10 genes (StHsp20-4, 6, 9, $10,11,14,30,36,44$ and 46) were not sensitive to salt and drought stresses, respectively. The remaining StHsp20s were up-regulated under salt and drought stresses, but the changes were not as extreme as that under heat stress. The differential expression patterns compared with those under heat stress indicated there were different response and regulatory mechanisms of the StHsp20 family under various abiotic stress conditions.

RNA-seq data of StHsp 20 under abiotic stress after treated for $24 \mathrm{~h}$ was collected from PGSC and processed to compare the expression abundance with that of qRT-PCR. The relative expression level was represented by stress/control (Additional file 4: 

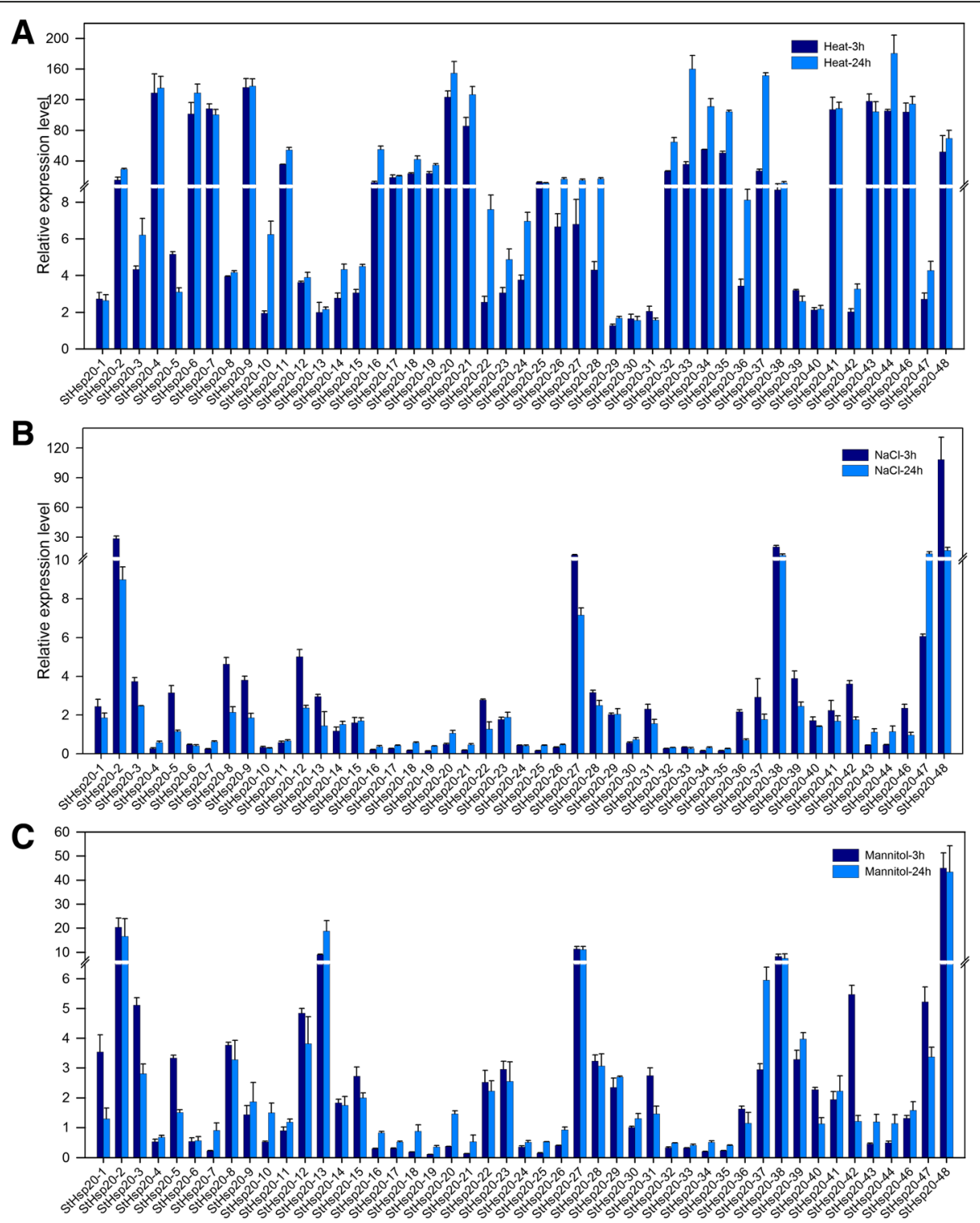

Fig. 6 Expression profiles of StHsp20 genes under heat, salt and drought stresses. Quantitative RT-PCR was used to investigate the expression levels of each StHsp20 gene. To calculate the relative expression level, the expression of each gene under control treatment was set as 1. The results were represented by mean \pm standard deviation. The reference gene used in qRT-PCR was efla

Figure S1). However, two sections of the results were not completely in accordance with each other. Under heat stress, only 10 of the StHsp20 genes showed high expression level while 20 StHsp20s showed low expression level. High expression levels were confirmed in nearly half of StHsp2Os under salt and drought stresses, meanwhile 3 and 2 StHsp2Os exhibited low expression level respectively. In a word, compared the two set of results from RNA-seq and qRT-PCR, 4 genes (StHsp20-5, 10, 13 and 22) showed a similar expression pattern under 3 abiotic stresses, and 5 genes (StHsp20-7, 15, 29, 42 and 47) had similar expression pattern under salt and drought stress.

\section{Discussion}

Hsp20s, as molecular chaperone, inhibit the irreversible aggregation of denaturing proteins, thus enhance the thermotolerance of plant [16]. With the availabilities of the whole genome sequence of many plants, several Hsp20 families have been identified, such as Arabidopsis, rice, Populus, pepper and tomato [23, 26, 27, 36]. However, little is known about Hsp20 family in potato.

The current study identified 48 StHsp 20 genes, and analyzed their structure, chromosomal location, phylogeny, gene duplication, stress-related cis-elements and expression patterns in different tissues and abiotic stresses. The study provides comprehensive information 
on the StHsp2O gene family and will aid in understanding the functional divergence of $H s p 20$ genes in potato.

Previous research identified 19, 39, 35 and $42 \mathrm{Hsp} 20$ genes in Arabidopsis, rice, pepper and tomato, respectively [24, 26, 27, 35]. The low number of $H s p 20$ genes in Arabidopsis is related to its small genome. Forty-eight Hsp 20 genes were identified in potato, which was close to the numbers found in pepper and tomato, which also belong to Solanaceae.

Gene organization plays a vital role in the evolution of multiple gene families [43]. In this study the percentage of intronless StHsp2O genes is similar to that of pepper (45.71\%) [26] and tomato (30.95\%) [27]. Additionally, StHsp 20 genes of the CII and ER subfamilies, as well as most StHsp2O genes of the CI subfamily, were intronless (Fig. 2, Table 1). Members of the CV, CVI, MI, MII, P and Po subfamilies had only one intron. The results are also in accordance with that in pepper and tomato. Additionally, similar motif arrangements were found in the same subfamily members (Figs. 1c, 2). This correlation between intron numbers and motif arrangement further confirmed the classifications of the StHsp20 genes. In some studies, genes with few or no introns were considered to have enhanced expression levels in plants [44, 45]. To response to various stresses timely, genes must be rapidly activated, which would be assisted by a compact gene structure with less introns [46]. Most of the StHsp20 genes were highly induced under heat stress (Fig. 6), which may approve the above standpoints in other research.

In earlier studies, Arabidopsis Hsp20 genes were classified into seven subfamilies (CI, CII, CIII, M, P, ER and Po), and five genes could not be clustered into any subfamily [23]. Subsequently, four new nucleocytoplasmic subfamilies (CIV, CV, CVI and CVII) and a mitochondrial subfamily (MII) were identified [35]. In our study, the phylogenetic tree showed that Hsp20 genes were classified into 12 distinct subfamilies. The StHsp 20 genes existed in 11 of the 12 subfamilies. There was no Hsp2O gene of potato in the CIV subfamily, which may be the result of gene loss during evolution.

Most of the StHsp2O genes (61.7\%) were grouped into a nucleocytoplasmic subfamily, which was also illustrated in Arabidopsis, pepper and tomato [23, 26, 27]. Among these subfamilies, CI was the largest subfamily, containing 18 StHsp 20 genes. Based on these results, we inferred that, because proteins are mainly synthesized in the cytoplasm, this could be the primary place for Hsp20 proteins to interact with denatured proteins, preventing inappropriate aggregation and degradation. Furthermore, the Hsp2O genes in the same subfamily from different species were more similar than those of the same species but belonging to various subfamilies. The finding indicated that synteny might exist in
Arabidopsis, Populus, rice and soybean Hsp20 proteins, and that Hsp20 subfamilies diversified before the divergence within these species.

The expansions of gene families and genome evolutionary mechanisms mainly depend on gene duplication events [47]. The major duplication patterns are tandem duplication and segmental duplication [48]. In this research, 48 StHsp 20 genes were located unevenly on 12 potato chromosomes, and most of the StHsp2O genes were located on the terminal regions of the chromosomes. Although the genome size of potato is almost 7 times that of Arabidopsis, the number of $H s p 20$ genes in potato (48 genes) is only 2.5 times that in Arabidopsis (19 genes). This could be the result of different whole genome duplication events in Arabidopsis and potato. A total of 21 StHsp2O duplicated genes were detected in potato, including one pair of segmentally duplicated genes (StHsp20-15 and StHsp20-48) and four tandem duplicated gene groups (Fig. 3), which revealed that both tandem and segmental duplications contributed to the evolution of $H s p 20$ genes in potato. Similar expression patterns under various abiotic stresses were found within the tandem duplicated gene groups (Fig. 6). The similar expression patterns indicated the analogous functions and structures of tandem duplicated StHsp2O genes. The redundancies of functions and similarities of structures may reflect shared induction mechanisms.

The expression patterns of $H s p 20$ genes in different tissues have been described in many species, such as Arabidopsis, rice, pepper and tomato [24, 26, 27, 35]. There is no uniform gene expression pattern for plant Hsp 20 genes. According to the RNA-seq data of potato, several StHsp2O genes such as StHsp20-22 and StHsp2041 , exhibited incongruous expression patterns in various tissues, indicating that different StHsp20 proteins may have diverse functions. Three genes, StHsp20-18, StHsp20-26 and StHsp20-30, were highly and indiscriminately expressed in all of the investigated tissues under normal condition. Similar with several $H s p 20$ genes in soybean, the three StHsp20s showed specific housekeeping expression activity [25].

qRT-PCR was used to investigate the transcript levels of each $S t H s p 20$ under different abiotic stresses. The two genes (StHsp20-29 and StHsp20-30) with distinctive expression patterns were highly expressed in all of the investigated tissues, but no induction was observed under heat stress. Thus, we may assume that the two genes are lacking of chaperone activities. The results confirmed the association of potato Hsp20 proteins with thermotolerance; however, the existence of numerous Hsp20s may lead to functional redundancy [6]. In addition, similar expression patterns in $S t H s p 20$ genes may be caused by shared induction mechanisms. Because the heat shock response network involves heat shock proteins and heat 
shock transcription factors (Hsfs), the expression levels of $H s p 2 O$ genes rely heavily on the activation of Hsfs under heat stress. During a 24-h heat treatment, the StHsp 20 genes showed different transcript accumulation levels. It was reported that the same set of Hsps could be regulated by different Hsfs on transcription level [49, 50], which indicated that $S t H s p 2 O$ genes are specifically controlled by various Hsfs. The differences in transcription levels of $S t H s p 20 s$ may be the reflection of different upstream regulating genes of $H s f s$.

Based on qRT-PCR, all of the StHsp2O genes responded to salt and drought stress; however, the expression level of several StHsp20s was down-regulated (Fig. 6). Under heat stress, Hsfs are activated and bound to HSEs in the Hsp2O gene promoters to regulate the expressions of downstream genes. Nevertheless, various cis-elements were found in promoter regions of StHsp2Os (Fig. 4), and these are involved in the responses of StHsp2O genes to other abiotic stresses. Thus, StHsp 20 genes could be induced by both heat stress and other abiotic stresses. The multiple abiotic stress responses of StHsp2O genes reflected an interconnected induction mechanism involving $H s f$ transcription factors.

Compared with expression pattern represented by RNA-seq data, the expression profile generated by qRTPCR was not completely equal to that. The difference of expression pattern may be caused by multiple reasons. Although the same plant material (DM) was used for research, only aboveground part of plant was collected in our research, while the whole plant was sampled for RNA sequencing. Specific to heat stress, the plant was treated for 24-h in normal photoperiod of $16 \mathrm{~h}$ light $/ 8 \mathrm{~h}$ dark in our study, but the plant for RNA sequencing was treated in the dark. The potato RNA-seq data used in our research was presented as FPKM. Compared with raw read counts, FPKM value can better reduce sample differences. However, the FPKM value could be significantly changed due to highly expressed genes [51]. The bias of FPKM value leads to different expression compared with qRT-PCR.

\section{Conclusions}

Here, a genome-wide analysis of potato $H s p 20$ family was performed, and $48 \mathrm{StHsp} 20$ genes were confirmed. Subsequently, analyses of $S t H s p 20$ genes on gene structures, phylogeny, chromosomal location, gene duplication, stress-related cis-elements, expression patterns in different tissues and abiotic stresses, were conducted based on bioinformatics and qRT-PCR methods. Most of StHsp2O genes were sensitive to heat stress and were up-regulated rapidly, indicating that $S t H s p 20$ genes play important roles in the acquired thermotolerance of potato. The study provides comprehensive information on the StHsp2O gene family in potato and will aid in determining the StHsp2O gene functions.

\section{Additional files}

Additional file 1: Table S1. The IDs of Hsp20 genes from Arabidopsis, soybean, rice and Populus. (DOCX $28 \mathrm{~kb}$ )

Additional file 2: Table S2. FPKM values of 48 StHsp2O genes in various potato tissues. (XLSX $15 \mathrm{~kb}$ )

Additional file 3: Table S3. The primer sequences of $48 \mathrm{StH}$ sp2O genes used for qRT-PCR. (DOCX $15 \mathrm{~kb}$ )

Additional file 4: Figure S1. Heatmap of StHsp2Os under heat, salt and drought stress. (TIFF $327 \mathrm{~kb}$ )

\section{Abbreviations}

AA: Amino acid; ABRE: ABA responsive element; ACD: Alpha-crystallin domain; BLASTP: Basic local alignment search tool-protein; CRI: Conserved region I; CRII: Conserved region II; DRE: Dehydration-responsive element;

FPKM: Fragments per kilobase of transcript per million mapped reads; GSDS: Gene structure display server; HMM: Hidden markov model; HSE: Heat stress element; Hsfs: Heat shock transcription factors; Hsps: Heat shock proteins; LTRE: Low temperature responsive element; MW: Molecular weight; ORF: Open reading frame; PGDD: Plant genome duplication database; PGSC: Potato genome sequencing consortium; PI: Isoelectric point; qRT-PCR: Quantitative real-time polymerase chain reaction; sHsp: Small heat shock proteins

\section{Acknowledgements}

This work was mainly funded by the National Natural Science Foundation of China (31500159) and partially supported by State Key Laboratory of Crop Stress Biology in Arid Areas, China. The funders had no role in study design, data collection and analysis, decision to publish, or preparation of the manuscript. We would like to thank the anonymous reviewers for their constructive comments.

\section{Funding}

This research was supported by the National Natural Science Foundation of China (31500159), Science and Technology Innovation Program of Agriculture Department of Shaanxi Province (2016CXY-05), the Natural Science Foundation of Shaanxi Province (2016JQ3029), and Undergraduate Innovation Foundation of Northwest A\&F University (1201610712119).

\section{Availability of data and materials}

All of the datasets supporting the results of this article are included within the article and its Additional files.

\section{Authors' contributions}

PZ and DDW collected the public dataset, perform bioinformatics analysis and also drafted the manuscript. RQW contributed to bioinformatics analysis and the making of all the figures and tables. NNK, CZ and $\mathrm{CHY}$ contributed to data collection. PZ, NNK and WTW performed the experiments. HLM and QC conceived this study and reviewed the manuscript. All of the authors read and approved the final manuscript.

Ethics approval and consent to participate Not applicable

\section{Consent for publication}

Not applicable

\section{Competing interests}

The authors declare that they have no competing interests.

\section{Publisher's Note}

Springer Nature remains neutral with regard to jurisdictional claims in published maps and institutional affiliations.

\section{Author details}

'State Key Laboratory of Crop Stress Biology for Arid Areas, College of Agronomy, Northwest A \& F University, Yangling, Shaanxi 712100, China. ${ }^{2}$ Innovation Experimental College, Northwest A \& F University, Yangling, Shaanxi 712100, China. 
Received: 28 March 2017 Accepted: 10 January 2018

Published online: 18 January 2018

\section{References}

1. Giorno F, Wolters-Arts M, Grillo S, Scharf KD, Vriezen WH, Mariani C. Developmental and heat stress-regulated expression of HSFa2 and small heat shock proteins in tomato anthers. J Exp Bot. 2009;61(2):453-62.

2. Wang W, Vinocur B, Altman A. Plant responses to drought, salinity and extreme temperatures: towards genetic engineering for stress tolerance. Planta. 2003;218(1):1-14.

3. Mittler R. Abiotic stress, the field environment and stress combination. Trends Plant Sci. 2006;11(1):15-9.

4. Herman DJ, Knowles LO, Knowles NR. Heat stress affects carbohydrate metabolism during cold-induced sweetening of potato (Solanum tuberosum L.). Planta. 2017;245(3):563-82.

5. De MA. Heat shock proteins: facts, thoughts, and dreams. Shock. 1999;11(1):1.

6. Waters ER. The evolution, function, structure, and expression of the plant sHsps. J Exp Bot. 2013;64(2):391

7. Mogk A, Bukau B. Role of sHsps in organizing cytosolic protein aggregation and disaggregation. Cell Stress Chaperon. 2017;22(4):493-502.

8. Wang W, Vinocur B, Shoseyov O, Altman A. Role of plant heat-shock proteins and molecular chaperones in the abiotic stress response. Trends Plant Sci. 2004;9(5):244-52.

9. Sarkar NK, Kim YK, Grover A. Rice sHsp genes: genomic organization and expression profiling under stress and development. BMC Genomics. 2009;10(1):393.

10. Waters ER, Lee GJ, Vierling E. Evolution, structure and function of the small heat shock proteins in plants. J Exp Bot. 1996;47(3):325-38.

11. Sung DY, Kaplan F, Lee KJ, Guy CL. Acquired tolerance to temperature extremes. Trends Plant Sci. 2003;8(4):179-87.

12. Vierling E. The roles of heat shock proteins in plants. Annu Rev Plant Biol. 2003;42(1):579-620.

13. Charng YY, Liu HC, Liu NY, Hsu FC, Ko SS. (2006). Arabidopsis Hsa32, a novel heat shock protein, is essential for acquired thermotolerance during long recovery after acclimation. Plant Physiol. 2006;140(4):1297-305.

14. Lee GJ, Vierling E. A small heat shock protein cooperates with heat shock protein 70 systems to reactivate a heat-denatured protein. Plant Physiol. 2000;122(1):189-98.

15. Cashikar AG, Duennwald M, Lindquist SL. A chaperone pathway in protein disaggregation. Hsp26 alters the nature of protein aggregates to facilitate reactivation by Hsp104. J Biol Chem. 2005;280(25):23869-75.

16. Haslbeck M, Vierling E. A first line of stress defense: small heat shock proteins and their function in protein homeostasis. J Mol Biol. 2015;427(7):1537-48.

17. Kirschner M, Winkelhaus S, Thierfelder JM, Nover L. Transient expression and heat-stress-induced co-aggregation of endogenous and heterologous small heat-stress proteins in tobacco protoplasts. Plant J. 2000;24(3):397-412.

18. Giese KC, Vierling E. Mutants in a small heat shock protein that affect the oligomeric state. Analysis and allele-specific suppression. J Biol Chem. 2004; 279(31):32674-83.

19. Basha $\mathrm{E}$, Friedrich $\mathrm{KL}$, Vierling E. The N-terminal arm of small heat shock proteins is important for both chaperone activity and substrate specificity. J Biol Chem. 2006;281(52):39943.

20. Jaya N, Garcia V, Vierling E, Lorimer GH. Substrate binding site flexibility of the small heat shock protein molecular chaperones. Proc Natl Acad Sci U S A. 2009;106(37):15604-9.

21. Bondino HG, Valle EM, Have AT. Evolution and functional diversification of the small heat shock protein/a-crystallin family in higher plants. Planta. 2012;235(6):1299-313.

22. Basha E, O'Neill H, Vierling E, et al. Trends Biochem Sci. 2012;37(3):106.

23. Scharf KD, Siddique M, Vierling E. The expanding family of Arabidopsis thaliana small heat stress proteins and a new family of proteins containing alpha-crystallin domains (acd proteins). Cell Stress Chaperon. 2001;6(3):225.

24. Ouyang Y, Chen J, Xie W, Wang L, Zhang Q. Comprehensive sequence and expression profile analysis of hsp20 gene family in rice. Plant Mol Biol. 2009; 70(3):341-57.

25. Lopes-Caitar VS, Carvalho MCD, Darben LM, Kuwahara MK, Nepomuceno AL, Dias WP, Abdelnoor RV, Marcelino-Guimarães FC. Genome-wide analysis of the Hsp20 gene family in soybean: comprehensive sequence, genomic organization and expression profile analysis under abiotic and biotic stresses. BMC Genomics. 2013;14(1):577.
26. Guo M, Liu JH, Lu JP, Zhai YF, Wang H, Gong ZH, Wang SB, Lu MH. Genome-wide analysis of the CaHsp20 gene family in pepper: comprehensive sequence and expression profile analysis under heat stress. Front Plant Sci. 2015;6:806.

27. Yu J, Cheng Y, Feng K, Ruan M, Ye Q, Wang R, Li Z, Zhou G, Yao Z, Yang Y, Wan $\mathrm{H}$. Genome-wide identification and expression profiling of tomato Hsp20 gene family in response to biotic and abiotic stresses. Front Plant Sci. 2016;7:1215.

28. Momčilović I, Pantelić D, Zdravković-Korać S, Oljača J, Rudić J, Fu J. (2016). Heat-induced accumulation of protein synthesis elongation factor 1a implies an important role in heat tolerance in potato. Planta. 2016;244(3):671-9.

29. Childs KL, Cepela J, Crisovan E. Spud db: a resource for mining sequences, genotypes, and phenotypes to accelerate potato breeding. Plant Genome. 2014;7(1):93-113.

30. Hu B, Jin J, Guo AY, He Z, Luo J, Gao G. GSDS 2.0: an upgraded gene feature visualization server. Bioinformatics. 2015;31(8):1296-7.

31. Voorrips RE. Mapchart: software for the graphical presentation of linkage maps and qtls. J Hered. 2002;93(1):77-8.

32. Gu Z, Cavalcanti A, Chen FC, Bouman P, Li WH. Extent of gene duplication in the genomes of drosophila, nematode, and yeast. Mol Biol Evol. 2002; 19(3):256-62.

33. Yang S, Zhang $X$, Yue JX, Tian D, Chen JQ. Recent duplications dominate nbs-encoding gene expansion in two woody species. Mol Gen Genomics. 2008;280(3):187.

34. Wang L, Guo K, Li Y, Tu Y, Hu H, Wang B, Cui X, Peng L. Expression profiling and integrative analysis of the cesa/csl superfamily in rice. BMC Plant Biol. 2010;10(1):282.

35. Siddique M, Gernhard S, Koskulldöring PV, Vierling E, Scharf KD. The plant sHsp superfamily: five new members in Arabidopsis thaliana with unexpected properties. Cell Stress Chaperon. 2008;13(2):183.

36. Waters ER, Aevermann BD, Sanders-Reed Z. Comparative analysis of the small heat shock proteins in three angiosperm genomes identifies new subfamilies and reveals diverse evolutionary patterns. Cell Stress Chaperon. 2008;13(2):127-42.

37. Thompson JD, Gibson TJ, Plewniak F, Jeanmougin F, Higgins DG. The clustal_x windows interface: flexible strategies for multiple sequence alignment aided by quality analysis tools. Nucleic Acids Res. 1997;25(25): 4876-82.

38. Tamura K, Stecher G, Paterson D, Filipski A, Kumar S. Mega6: molecular evolutionary genetics analysis software version 6.0. Mol Biol Evol. 2007;24(8):1596-9.

39. Lescot M, Déhais $P$, Thijs G, Marchal K, Moreau Y, Yves VDP, Pieree R, Stephane R. Plantcare, a database of plant cis-acting regulatory elements and a portal to tools for in silico analysis of promoter sequences. Nucleic Acids Res. 2002;30(1):325-7.

40. Murashige T, Skoog F. A revised medium for rapid growth and bio assays with tobacco tissue cultures. Physiol Plant. 2006;15(3):473-97.

41. Deng W, Wang Y, Liu Z, Cheng H, Xue Y. Hemi: a toolkit for illustrating heatmaps. PLoS One. 2013;9(11):e111988.

42. Cannon SB, Mitra A, Baumgarten A, Young ND, May G. The roles of segmental and tandem gene duplication in the evolution of large gene families in Arabidopsis thaliana. BMC Plant Biol. 2004:4:10.

43. Xu G, Guo C, Shan H, Kong H. Divergence of duplicate genes in exon-intron structure. Proc Natl Acad Sci U S A. 2012;109(4):1187-92.

44. Ren X, Vorst O, Fiers M, Stiekema WJ, Nap J. In plants, highly expressed genes are the least compact. Trends Genet. 2006;22(10):528-32.

45. Chung BY, Simons C, Firth AE, Brown CM, Hellens RP. Effect of 5'utr introns on gene expression in Arabidopsis thaliana. BMC Genomics. 2006;7(1):120.

46. Jeffares DC, Penkett CJ, Bähler J. (2008). Rapidly regulated genes are intron poor. Trends Genet. 2008;24(8):375-8.

47. Vision TJ, Brown DG, Tanksley SD. (2000). The origins of genomic duplications in Arabidopsis. Science. 2000;290(5499):2114-7.

48. Kong $\mathrm{H}$, Landherr LL, Frohlich MW, Leebensmack J, Ma H, Depamphilis CW. Patterns of gene duplication in the plant skp1 gene family in angiosperms: evidence for multiple mechanisms of rapid gene birth. Plant J. 2007;50(5):873-85.

49. Schramm F, Ganguli A, Kiehlmann E, Englich G, Walch D, Koskull-Döring PV. The heat stress transcription factor $\mathrm{Hsfa2}$ serves as a regulatory amplifier of a subset of genes in the heat stress response in Arabidopsis. Plant Mol Biol. 2006;53(5):264.

50. Tang R, Zhu W, Song X, Lin X, Cai J, Wang M, Yang Q. Genome-wide identification and function analyses of heat shock transcription factors in potato. Front Plant Sci. 2016;7(7):490.

51. Kukurba KR, Montgomery SB. RNA sequencing and analysis. Cold Spring Harb Protoc. 2015;11:951-69. 